\title{
Olympische Erziehung und Bildung in Deutschland: Ansätze der Deutschen Olympischen Akademie
}

\author{
Gerald Fritz, Matthias Thaler
}

Das Spektrum an Perspektiven, das dieser Sammelband anbietet, ist bewusst sehr breit angelegt, ohne einen Anspruch auf Vollständigkeit erheben zu wollen. Wenn man, ausgehend von dieser Fülle an Themen, nun Ansatzpunkte für die olympische Bildungsarbeit identifizieren möchte, ist man geneigt, Thomas Röhlers Ausführungen in dieser Publikation zuzustimmen: Die Zeit der Wettkämpfe, ob nun Olympische Spiele, Paralympics, Deaflympics oder Special Olympics World Games, ist dafür nur bedingt geeignet. Ein Rahmenprogramm kann zwar gewisse Tendenzen aufgreifen und einzelne Schwerpunkte in den Fokus rücken, die Aufmerksamkeit aller Beteiligten richtet sich in diesen Tagen aber in erster Linie auf das sportliche Geschehen.

Die Bildungsarbeit muss sich daher auf die Zeit zwischen den Spielen, während der Olympiade, konzentrieren und von der nationalen Ebene ausgehen. Die Olympische Charta schreibt jedem NOK vor, sich für die Verbreitung der Olympischen Idee einzusetzen und Programme im Bereich der Olympischen Erziehung zu fördern.

Der DOSB, der in Deutschland als NOK fungiert, stellte die zentrale Bedeutung dieser Aufgabe bei der Gründung der Deutschen Olympischen Akademie Willi Daume e. V. im Mai 2007 heraus. Als Nachfolgeinstitution des Deutschen Olympischen Instituts und des Kuratoriums Olympische Akademie und Olympische Erziehung trägt die DOA die Verantwortung für die Verbreitung der Olympischen Idee in Deutschland und setzt zahlreiche Maßnahmen für verschiedene Zielgruppen um.

Im Bereich der Olympischen Erziehung etwa sind die „Olympia ruft: Mach mit!"-Unterrichtsmaterialien seit Ende der 1980er Jahre etabliert. $\mathrm{Zu}$ Olympischen Spielen erscheinen jeweils spezifische Broschüren und Online-Materialien für verschiedene Altersstufen, die thematisch an das jeweilige Gastgeberland und dessen Kultur anknüpfen. Fächerübergreifende Unterrichtsentwürfe und Arbeitsblätter liefern Lehrkräften vielfältige Anregungen, um olympische Themen im Unterricht zu behandeln. Die Materialien sind online und kostenlos zugänglich, um möglichst viele Bildungseinrichtungen mit diesem Angebot zu erreichen. 
In Fortbildungen werden diese Ansätze an Lehrkräfte vermittelt. In zweijährigem Rhythmus wird eine Lehrerfortbildung in Griechenland durchgeführt, die bereits seit den frühen 90er Jahren Vorträge und Workshops mit einem Bildungsprogramm verknüpft. Der Kern der Fortbildung findet dabei auf dem Gelände der Internationalen Olympischen Akademie (IOA) in Olympia statt, dem internationalen Studienzentrum der Olympischen Bewegung. Seit 2019 wird zudem in Kooperation mit der Nationalen Anti-Doping Agentur Deutschland (NADA) eine Fortbildung zu den Themen Dopingprävention und Werteerziehung umgesetzt.

Die Jugendlager anlässlich Olympischer Spiele richten sich gezielt an hoffnungsvolle Nachwuchsleistungsportler*innen und ehrenamtlich im Sport engagierte Jugendliche. Eingebettet in ein Bildungs- und Kulturprogramm, das gemeinsam mit der Deutschen Sportjugend als Partner organisiert wird, bieten sie die Möglichkeit, die Wettkämpfe und die besondere Atmosphäre vor Ort im Gastgeberland zu erleben.

Einen erlebnisorientierten Zugang zu olympischen Themen bietet der Olympic Day, der die Gründung des IOC in Erinnerung ruft. Kinder und Jugendliche können bei dieser eintägigen Veranstaltung Sportarten praktisch ausprobieren und sich an Infoständen mit Themen wie gesunder Ernährung oder Dopingprävention auseinandersetzen. Seit 2020 wurde das Programm über die Präsenzveranstaltung in Köln hinaus erweitert. Am virtuellen Olympic Day Run, der im Jahr 2020 ins Leben gerufen wurde, nehmen Menschen jeden Alters aus ganz Deutschland teil. Außerdem beteiligen sich seit einigen Jahren mit wachsender Tendenz auch verschiedene Hochschulen und Universitäten mit akademischen Programmpunkten am Olympic Day.

Darüber hinaus bildet seit mehr als zwei Jahrzehnten das Sportwissenschaftliche Olympiaseminar einen Schwerpunkt in der akademischen Bildungsarbeit der DOA. Universitäten und Hochschulen aus ganz Deutschland können sich um die Teilnahme bewerben. Ziel dieser Veranstaltung ist es, einen interdisziplinären Diskurs über historische und aktuelle Fragen der Olympischen Bewegung zu ermöglichen und einen intensiven Austausch zwischen Studierenden und Lehrenden aus den beteiligten akademischen Einrichtungen zu fördern.

Im Rahmen der Gesprächsreibe „Biebricher Schlossgespräche“ werden Fragestellungen aufgegriffen, die auch über den Sport hinaus gesellschaftliche Relevanz besitzen und von Expert*innen diskutiert werden. Das Format richtet sich an ein Fachpublikum aus Sport, Gesellschaft, Politik und Wirtschaft. Seit einigen Jahren ist auch die Verleibung des Fair Play Preises des Deutschen Sports in diese Veranstaltung eingebettet. 
Im internationalen Kontext schließlich prägt die enge Zusammenarbeit mit der IOA die Aktivitäten der DOA, die für die Auswahl und Vorbereitung der deutschen Teilnehmer*innen an den verschiedenen Sessionen verantwortlich ist. Im europäischen Kontext stellen die Beteiligung an verschiedenen Erasmus+ Projekten und die Zusammenarbeit mit dem Dachverband der European Olympic Academies (EOA), der seinen Sitz in Frankfurt hat, wichtige Säulen der Bildungsarbeit dar.

Darüber hinaus gibt es zahlreiche weitere Projekte, Initiativen und Kooperationen, mit deren Hilfe die DOA ihren satzungsgemäßen Bildungsauftrag verfolgt. Über verschiedene Kanäle werden sowohl die vorgestellten Maßnahmen und ihre Ergebnisse einer breiten Öffentlichkeit zugänglich gemacht als auch über einzelne Formate im Social-MediaBereich gezielt Inhalte vermittelt.

Den hier angesprochenen Ansatz Olympischer Bildung im Sinne einer ganzheitlichen und lebenslangen Auseinandersetzung mit olympischen Themen füllt die Deutsche Olympische Akademie mit diesen Aktivitäten also bereits jetzt mit Leben. Um die Olympische Idee weiterzuentwickeln und zu verbreiten, wird es künftig darauf ankommen, bestehende Ansätze mit neuen Innovationen zu verknüpfen. Dabei gilt es, vor allem die Möglichkeiten der zunehmenden Digitalisierung zu nutzen und aktuelle Themen der Olympischen Bewegung wissenschaftlich zu begleiten. Entscheidend wird sein, die Vernetzung mit Multiplikator*innen in allen gesellschaftlichen Bereichen zu intensivieren, um so dauerhaft die Wirksamkeit der Inhalte Olympischer Erziehung und Bildung auf verschiedenen Ebenen zu sichern. 
